\title{
Microwave Broadband Characterization of Aging of SU-8 Polymer as CPW Substrate
}

\author{
Andrea Lucibello, Giovanni Maria Sardi, Emanuela \\ Proietti, Romolo Marcelli \\ Institute for Microelectronics and Microsystems, \\ National Research Council of Italy (CNR-IMM) \\ Via del Fosso del Cavaliere 100, 00133 Rome, Italy \\ romolo.marcelli@artov.imm.cnr.it
}

\author{
Giancarlo Bartolucci \\ Department of Electronic Engineering, \\ University of "Roma Tor Vergata" \\ Via del Politecnico 1, 00133 Rome, Italy \\ bartolucci@eln.uniroma2.it
}

\begin{abstract}
In this paper we present the methodology and the numerical results related to the analysis of aging of the $\mathrm{SU}$ 8 polymer when used as a primary layer for the realization of Coplanar Waveguide (CPW) structures. As test devices, we used a set of transmission lines with different lengths and T-shaped open stubs shunt resonators; by using these geometries, we are able to acquire the data in a broadband range, in principle between $1 \mathrm{GHz}$ and $40 \mathrm{GHz}$. We conduct the analysis by comparing two different technology run: the first wafer with a deposited layer by a 12-year-old SU-8 and the second wafer, with the same photolithographed metallic geometries, with a brand-new processed $\mathrm{SU}-8$ photoresist.
\end{abstract}

Keywords-RF MEMS, SU-8, CPW, Aging, Microwave.

\section{INTRODUCTION}

Coplanar Waveguide (CPW) is one of the most used geometry for high frequency - high-speed transmission lines design in microwave electronics. Its complete planarity, with the signal line and grounds placed on the same plane, allows an easy integration and fabrication in ultra-large-scale process. At the same time, the negative epoxy photoresist SU-8 has been employed, now for 20 years, in the process of realization of Micro Electro Mechanical Systems (MEMS) sensors, microwave devices, optoelectronics and, lately, as a polymer for the realization of complex microfluidic channels [1-2]. SU-8 became popular also for the possibility to pattern structures with multiple levels and for managing geometries with high aspect ratio, like pillars, for example in MEMS [3] and a sensor array for biological application [4]. Because of the possibility to use SU-8 by means of MEMS technologies, low-loss CPWs have been also demonstrated on low resistivity silicon, exciting an almost TEM propagation [5-6]. Despite this well-deserved popularity, now it is clear that an accurate characterization in terms of electromagnetic properties is vital and crucial for an effective and efficient design of high frequency devices and, more in general, 3D structures. Suppliers quite often skip this kind of characterization, and designers must rely on some online scattered values with unverified reliability. Few previous works studied the behavior of the electrical permittivity of SU-8 in a broad frequency span. A first paper reports the data in the frequencies 1-4 GHz by using a resonant ring structure in a micro-strip configuration [7]; a second one used a conductor backed CPW [8] for conducting this analysis, with reliable results between $5 \mathrm{GHz}$ to $50 \mathrm{GHz}$; in a third one a micromachined strip line is buried by SU-8 [9] to extract its characteristic impedance, propagation constant and, by them, to derive the dielectric constant of the polymer. In this work, we characterized in terms of propagation constant and impedance [10-11] a set of 16 different CPW lines and 15 different Tshaped resonators (CPW lines with in parallel an open-ended stub [12]). S-parameters are acquired between $1 \mathrm{GHz}$ and 40 $\mathrm{GHz}, 801$ points, by ground-signal-ground (G-S-G) RF probes directly on-wafer. Data are then processed to extract the $a$ priori unknown electrical permittivity of the SU-8. In this way, we are able to extract the dielectric constant of SU-8 in a broadband range, and to verify the variation of the electromagnetic properties of the polymer after aging (12 years). The polymer aged still in liquid form, unprocessed, conserved in his original bottle and maintained in clean-room environment. This is very useful in view of the implementation of RF configurations implying SU-8 MEMS technology for both device and packaging purposes. The knowledge of material aging, and the correspondent change in dielectric properties and frequency matching of the devices manufactured on top of it, is a key factor especially for determining suitable space applications, where the change of material properties could result in big issues for the electrical response of devices based on the chosen technology. Polymers were typically avoided in some applications because of out-gassing phenomena, leading to leakage in case of hermetic packaging requirements for long-term performance. Moreover, plastic materials could be not stable over the years, and material properties can change. On the other hand, wellestablished protocols could fail in having a precise determination of the material aging, because they are normally based on thermal treatments and extrapolations from short-term heating and thermal shock procedures. In this work, we had the opportunity to characterize materials distinguished only by the age, but preserved in the same Laboratory, with controlled temperature and humidity boundary conditions, over the years. For the above reasons, the work is not systematic, as we had no SU-8 purposely aged for this study following a pre-defined strategy, but we guess it is in any case an important result to show the modifications introduced by natural aging, without any other worsening factor for the electrical performances. The 
paper is organized as follows: after this introduction, in Sec. II we describe the methodology adopted for this research, including theoretical main aspects. Then, in Sec. III we describe the experiments and discuss the results. In the end, conclusions are drawn.

\section{Methodology}

\section{A. Theoretical approach}

In this paper, for brevity, we do not recall the complete mathematical formulation adopted. For a complete review, the reader can refer to works [10] and [11]. The logical scheme describing the procedure for calculating the dielectric constant of the SU-8 layer is as follows: 1) acquire S-parameters as 2ports data on the test devices by broadband (1-40 GHz) on wafer measurements; 2 ) by using matrix transformation, we are able to compute the propagation constant $k=\beta-j \alpha$ on the transmission lines and to extract the resonant frequency $f_{r}$ for each T-resonator; from the extracted $\beta$ (phase constant) it is calculated the effective dielectric constant $\varepsilon_{\text {eff }}$ of the line (in the broadband regime), while from $f_{r}$ the $\varepsilon_{\text {eff }}$ is determined precisely at the resonant frequency; 3 ) in the end, by inverting design formulas on CPW [13] and T-resonator, the unknown dielectric constant $\varepsilon_{\mathrm{r}-\mathrm{SU} 8}$ of the polymeric layer is derived.

\section{B. Test devices}

The devices are realized by standard photolithography: starting from a high resistivity 4-inch Silicon wafer, $525 \mu \mathrm{m}$ thick, we then deposited a $25 \mu \mathrm{m}$ thick SU-8 2000 layer; in the end the metallization of the lines and resonators is patterned. By using the CPW lines we are able to process the data in a broadband regime, while the different resonances, characteristic of the T-geometries, allows us a more stable data on particular frequencies to be used for a later best fitting procedure. We realized two different test cases: in the first one, very aged SU8 (12 years) was processed and used as substrate for the above devices; in a second run the same geometries are realized on fresh SU-8, just shipped by the company. In Fig. 1 the layout of the test devices is presented, and in Tab. I, the three geometrical parameters of the four different lines are listed. For each typology, we have the first line $10 \mathrm{~mm}$ long, the second line 11 $\mathrm{mm}$ long and the third one $12 \mathrm{~mm}$. Having three lines with different length allows us the application of the differential analysis method presented in [9]. Regarding the T-stub resonator, the lengths of the stubs are designed as $3 \mathrm{~mm}, 4 \mathrm{~mm}$, $5 \mathrm{~mm}, 7 \mathrm{~mm}$, except for the Type 4 lines, where we used $6 \mathrm{~mm}$, $7 \mathrm{~mm}$ and $9 \mathrm{~mm}$. The expected resonances, i.e. $\mathrm{S}_{21}=0$ ideally, obey the following equation:

$$
f_{\text {res }}=\frac{n c_{0}}{4 l_{s t u b} \sqrt{\varepsilon_{e f f}}}
$$

Where, $n$ is the resonance order, $c_{0}$ is the speed of light in vacuum, $l_{\text {stub }}$ is the physical length of the shunt stub, and $\varepsilon_{\text {eff }}$ is the effective dielectric constant of a two-layered ungrounded CPW line, as in $[12,13]$.
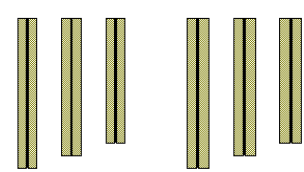

Type 1

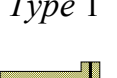

Type 2
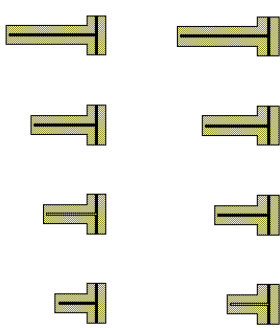

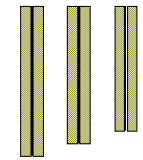

\section{Type 3}
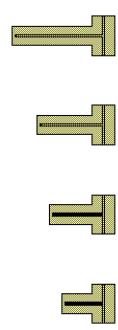

Type 4
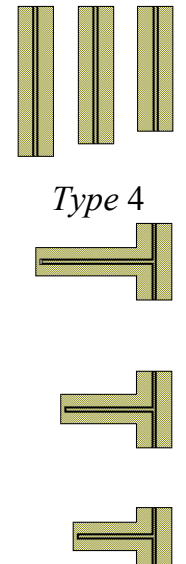

Fig 1. Layout of the test devices: from left to right, grouped in columns, Type 1, 2,3 , and 4 respectively lines geometry: transmission lines above and $\mathrm{T}$ resonators below.

TABLE I. Geometrical parameters of the lines grouped by typology (the table reports the width of the signal line, the air gap between signal line and lateral ground planes and the width of the ground planes)

\begin{tabular}{|r|c|c|c|c|}
\hline & Type 1 & Type 2 & Type 3 & Type 4 \\
\hline w_signal & $90 \mu \mathrm{m}$ & $100 \mu \mathrm{m}$ & $120 \mu \mathrm{m}$ & $250 \mu \mathrm{m}$ \\
\hline w_gap & $25 \mu \mathrm{m}$ & $30 \mu \mathrm{m}$ & $40 \mu \mathrm{m}$ & $100 \mu \mathrm{m}$ \\
\hline w_ground & $700 \mu \mathrm{m}$ & $800 \mu \mathrm{m}$ & $800 \mu \mathrm{m}$ & $1200 \mu \mathrm{m}$ \\
\hline
\end{tabular}



Fig 2. Photo of the processed wafer, test case 1.

\section{EXPERIMENT AND DISCUSSION OF RESULTS}

After the completion of both wafers, in Fig 1 is displayed test case 1 (test case 2 is visually identical), we started measuring each device on each wafer. In total, we have a set of 54 2-port measurements. The comparison of the dielectric constant values between fresh and aged SU-8 related to the transmission lines is depicted in Fig. 3. Due to a known limitation of the methodology, having designed short transmission lines, in the low frequency regime (below $5 \mathrm{GHz}$ ), the information of the dielectric constant is not accurate. We can compensate this inaccuracy by the analysis of the behavior of the resonators. 


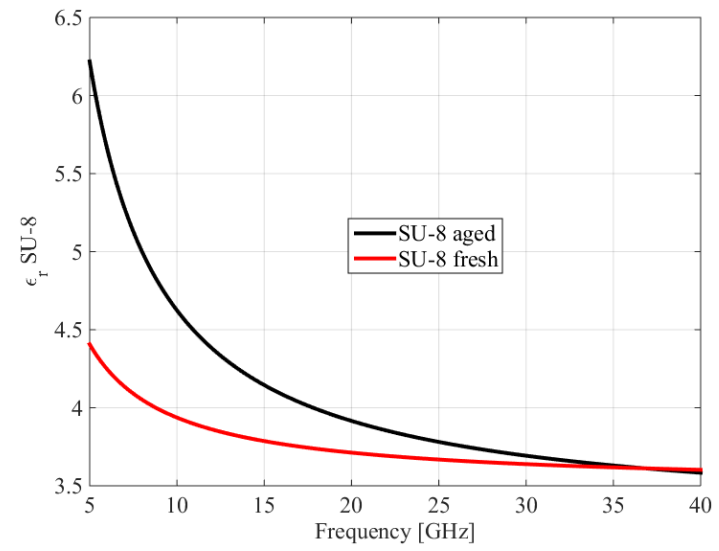

Fig 3. Extracted dielectric constant from the analysis of the transmission lines.

Regarding the data coming out by the analysis of the Tresonator, we identified clearly the first two resonances, respectively around $5.890 \mathrm{GHz}$ and $17.970 \mathrm{GHz}$. By inverting the design equation from [11], with a physical length of the stub of $7 \mathrm{~mm}$, we obtain the following data:

- $\quad \varepsilon_{\mathrm{r}} \mathrm{SU}-8$ fresh 3.4, SU-8 aged $3.8 @ 5.8 \mathrm{GHz}$

- $\varepsilon_{\mathrm{r}}$ SU-8 fresh 3.2, SU-8 aged 3.3@17.9 GHz

For a better understanding of what does it mean in terms of electrical performances of a planar device manufactured on a layer of thick SU-8, we can say, first of all, that such a change in the dielectric constant can cause a change in the characteristic impedance around $\Delta \mathrm{Z} \approx 1 \mathrm{ohm}$, and a corresponding variation of the return loss in the order of $3 \mathrm{~dB}$.

Looking to the frequency changes induced in a resonator, which is the most sensitive device to the dielectric constant modifications, we can also write, by using the definition of resonant frequency or from the cut-off: $f_{r} \sim 1 / \sqrt{\varepsilon}$, which corresponds to $f_{r}^{\prime} / f_{r}=\sqrt{\varepsilon^{\prime} / \varepsilon} \approx 1.05 @ 5.8 \mathrm{GHz}$ and $1.01 @$ $17.9 \mathrm{GHz}$. This simple computation gives evidence for a less critical aging contribution at higher frequencies, even if a change of $1 \%$ at $20 \mathrm{GHz}$ means a not negligible $200 \mathrm{MHz}$ shift.

To complete the characterization, we proceed further in the analysis of the losses. We want to verify if aging process changes in a noticeable way the microwave losses of the polymer. This parameter is also interesting for designers, who would like to know how the substrate changes in time, and for system engineers, to predict the whole RF system evolution. By a direct comparison of the $S_{21}$ parameter, or Insertion Loss (IL), measured between linear section of transmission lines, we can observe, Fig. 4, that is evident that aged SU8 is more lossy than fresh one in the whole frequency range analyzed. In this result, we are comparing $10 \mathrm{~mm}$ length lines, and we can summarize the observed difference of losses (see selected marker in figure):

$$
\begin{aligned}
& \text { - } \quad+0.038 \mathrm{~dB} \text { IL at } 5 \mathrm{GHz}(+2.6 \% \text { losses }) \\
& \text { - } \quad+0.078 \mathrm{~dB} \text { IL at } 10 \mathrm{GHz}(+4 \% \text { losses }) \text {; } \\
& \text { - } \quad+0.17 \mathrm{~dB} \text { IL at } 15 \mathrm{GHz}(+5 \% \text { losses } 20 \mathrm{GHz}(+8 \% \text { losses }) \text {; } \\
& \text { - } \quad+0.036 \mathrm{~dB} \text { IL at } 25 \mathrm{GHz}(+1 \% \text { losses }) \\
& \quad+0.641 \mathrm{~dB} \text { IL at } 30 \mathrm{GHz}(+22 \% \text { losses }) \text {. }
\end{aligned}
$$

Regarding the data at $30 \mathrm{GHz}$, and above this frequency, the results start to be influence by field leakage effects (radiation in free space), because the length of the lines is now comparable with a significant fraction of the wavelength. For this reason, in principle, we should shield all metallic equipment in the nearby of the wafer under test: mainly the RF probes chassis and the RF probe micro-positioners,

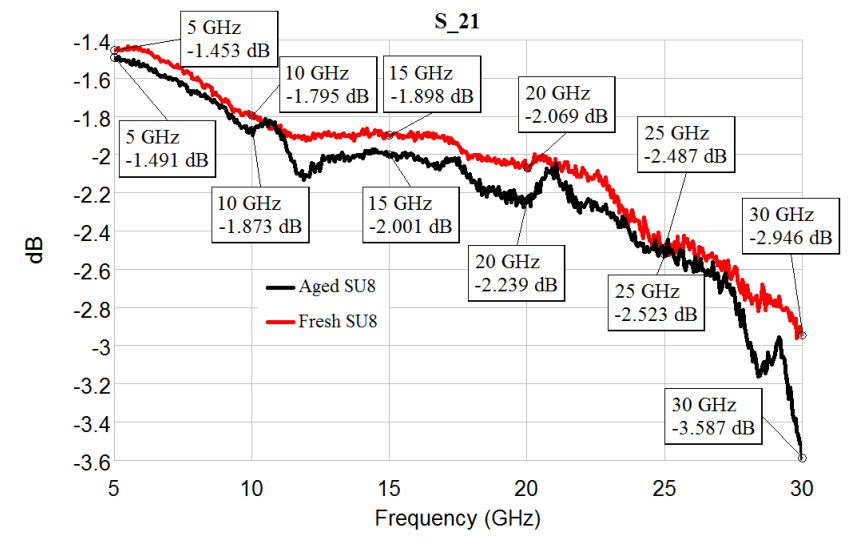

Fig 4. Comparison of losses on a unitary length line $(10 \mathrm{~mm})$ between aged and fresh SU-8.

\section{CONCLUSIONS}

The characterization of the aging of the SU-8 polymer has been performed by using it as primary layer for CPW lines and T-resonators in two different wafer run. By measuring the 2ports S-parameters of the different test structures we calculated the dielectric constant of the polymer and by comparison of the same structures on different wafers we verified the change in dielectric constant. From a complete analysis, we can observe that very aged SU-8 seems to have a slightly higher dielectric constant in broadband situation, probably due to very slow drying process experienced by the material during the natural aging. This condition is similar also regarding microwave losses induced by the polymer; in fact, the aged one seems to be more lossy with equal geometry, on average by $5 \%$ between 5 and 20 GHz. The possibility to use a naturally aged material, preserved in controlled environment over the years gives a prediction free from any extrapolation about the possible long-term utilization of such a polymer without using specific thermal treatments. In particular, a clear indication about the possibility to use SU-8 even after 10 years ca. without critical changes in the electrical performances of microwave planar devices is given.

\section{REFERENCES}

[1] A. Bertsch, P. Renaud, "Special Issue: 15 Years of SU8 as MEMS Material", Micromachines, Vol. 6, No. 6, 2015.

[2] H. Lorenz, M. Despont, N. Fahrni, N. La Bianca, P. Vettiger, P. Renaud, "SU-8: A low-cost negative resist for MEMS", Journal of Micromechanics and Microengineering, Vol. 7, No. 3, 121-124, 1997.

[3] E. H. Conradie, D. F. Moore: "SU-8 thick photoresist processing as a functional material for MEMS applications", Journal of Micromechanics and Microengineering, Vol. 12, No. 4, 368, 2002.J. C. Doll, N. Harjee, N. Klejwa, R. Kwon, S. M. Coulthard, B. Petzold, M. B. Goodmanc. B. L. Pruitt, "SU-8 force sensing pillar arrays for biological measurements", Lab on Chip, Vol, 9, No. 10, 1449-1454, 2009. 
[4] R. Marcelli, S. Catoni, L. Frenguelli: "Low Loss Coplanar Lines on Low Resistivity Silicon by SU-8 Thick Negative Photoresist"; $28^{\text {th }}$ International Semiconductor Conference, IEEE CAS 2005, October 3-5, 2005, Sinaia.

[5] A. Lucibello, E. Proietti, F. Giacomozzi, R. Marcelli, G. Bartolucci, G. De Angelis: "RF MEMS Switches Fabrication by using SU-8 Technology", Microsystem Technology, Vol. 6, No. 6, 929-936, 2013.

[6] J. M. Dewdney, J. Wang, "Characterization the microwave properties of SU-8 based on microstrip ring resonator", IEEE Wireless and Microwave Technology Conference, 2009. WAMICON '09, April 20-21, 2009.

[7] F. D. Mbairi, H. Hesselbom, "High frequency design and characterization of SU-8 based conductor backed coplanar waveguide transmission lines," International Symposium on Advanced Packaging Materials: Processes, Properties and Interfaces 2005, March 16-18, 2005.

[8] R. Osorio, M. Klein, H. Massler, J. G. Korvin, "Micromachined Strip Line with SU-8 as the Dielectric", Gallium Arsenide applications symposium. GAAS 2003, 6-10 October 2003, Munich.
[9] J. A. Reynoso-Hernández, C. F. Estrada-Maldonado, T. Parra, K. Grenier, J. Graffeuil, "An Improved Method for the Wave Propagation Constant $\gamma$ Estimation in Broadband Uniform Millimeter-Wave Transmission Line", Microwave and Optical Technology Letters, Vol. 22, No. 4, 268-271, 1999.

[10] J. Hinojosa, K. Lmimouni, S. Lepilliet, G. Dambrine, "Very High Broadband Electromagnetic Characterization Method of Film-Shaped Materials using Coplanar", Microwave and Optical Technology Letters, Vol. 33, No. 5, 352-355, 2002.

[11] R. L. Peterson, R. F. Drayton, "A CPW T-Resonator Technique for Electrical Characterization of Microwave Substrates", IEEE Microwave and Wireless Components Letters, Vol. 12, No. 3, 90-92, 2002.

[12] R. N. Simons, "Coplanar Waveguide Circuits, Components and Systems", Wiley-Interscience, 2001.

[13] S. S. Gevorgian, "Basic characteristics of two layered substrate coplanar waveguides", Electronics Letters, Vol. 30, No. 15, 1236-1237, 1994. 